\title{
Short communication: Genetic correlations between number of embryos produced using in vivo and in vitro techniques in heifer and cow donors
}

\author{
C. Jaton, ${ }^{*} \dagger^{1}$ A. Koeck, ${ }^{*}$ M. Sargolzaei, ${ }^{*} \dagger$ C. A. Price, $\ddagger$ C. Baes, ${ }^{*}$ F. S. Schenkel, ${ }^{*}$ and F. Miglior ${ }^{\star} \S$ \\ *Centre for Genetic Improvement of Livestock (CGIL), University of Guelph, Guelph, Ontario, Canada, N1G 2W1 \\ †The Semex Alliance, Guelph, Ontario, Canada, N1G $3 Z 2$ \\ łUniversité de Montréal, Faculté de médecine vétérinaire, St-Hyacinthe, Québec, Canada, J2S 2M2 \\ §Canadian Dairy Network (CDN), Guelph, Ontario, Canada, N1K 1E5
}

\begin{abstract}
Multiple embryos can be produced from a heifer or cow donors using an in vivo or an in vitro technique. Comparisons of the number of embryos produced by the same donors as heifers and cows and using different techniques are limited. The main objectives of this study were to assess the genetic correlation between the number of embryos produced by Holstein donors using an in vivo and in vitro technique as a heifer and as a cow. The data set used was recorded by Holstein Canada and included all successful superovulations or ovum pickup and in vitro fertilization procedures performed on Holstein donors for more than $20 \mathrm{yr}$. The type of technique used was known for all records and the status of the donor at recovery was retrieved from calving records. Bivariate repeatability animal model analyses were performed for both the total number of embryos (NE) and the number of viable embryos (VE) recovered per procedure. Logarithmic transformation was performed on the traits to normalize the data. Heritability estimates for the donor varied between 0.14 (0.02) and 0.19 (0.03) over all analyses, indicating that the number of embryos produced by a donor is influenced by the genetic potential of the donor. Genetic correlations between records produced in vivo and in vitro were moderately high and positive $(\mathrm{NE}=0.85 \pm$ $0.07 ; \mathrm{VE}=0.63 \pm 0.09$ ), suggesting that donors with high genetic potential for in vivo superovulation tend also to have high potential to produce multiple embryos in vitro. Similarly, the moderately high genetic correlations $(\mathrm{NE}=0.79 \pm 0.05 ; \mathrm{VE}=0.72 \pm 0.05)$ found between heifer and cow records indicate that a donor tends to produce a comparable number of embryos as a heifer or as a cow. The estimated repeatabilities (0.23 to $0.35)$ indicated that the number of embryos recovered should be somewhat repeatable in the same donor over
\end{abstract}

Received April 24, 2016.

Accepted July 6, 2016.

${ }^{1}$ Corresponding author: cjaton@uoguelph.ca time. On the other hand, the service sires seem not to play an important role on the total number of embryos produced by a donor no matter the technique used or the status of the donor at recovery.

Key words: superovulation, OPU-IVP, heifer, cow

\section{Short Communication}

Genetic gain in a population can be improved by producing more embryos from elite females. Multiple embryos can be produced using 2 different techniques, either using superovulation and producing the embryos in vivo or using ovum pickup (OPU) and in vitro production (IVP) of the embryos. Both techniques and their many protocols have been described very well by many authors since the commercial availability of multiple ovulation and embryo transfer in the 1970s (Farin et al., 2007; Machaty et al., 2012; Hasler, 2014). However, comparisons of the number of embryos produced by different techniques in the same donors are limited. To our knowledge, no study has estimated genetic correlation between the number of embryos produced in vivo and in vitro for purebred Bos taurus cattle donors. Moreover, embryos can be produced by donors cows or virgin heifers as young as 7 mo of age (e.g., www. boviteq.com) using either of the 2 techniques. Most fertility traits in dairy cattle in Canada are evaluated separately for heifers and for cows because of the different heritabilities estimated for these 2 groups and because the genetic correlation between fertility traits in heifers and cows is below one (Jamrozik et al., 2005). Considering that embryo production is also a reproduction trait, we were interested in comparing the genetic differences between heifers and cows for the number of embryos produced. To our knowledge, no study has assessed the genetic correlation between number of embryos produced by donors as both heifers and cows. Therefore, the objectives of the current study were (1) to assess the genetic correlation between the number of embryos produced in vivo and in vitro by Holstein donors, (2) to evaluate the genetic correlation of the 
Table 1. Number of records, number of donors, mean total number of embryos (NE), and mean number of viable embryos (VE) for different techniques and donors (standard deviation)

\begin{tabular}{lrrccc}
\hline Item & $\begin{array}{r}\text { No. of } \\
\text { records }\end{array}$ & $\begin{array}{c}\text { No. of } \\
\text { donors }\end{array}$ & $\begin{array}{c}\text { No. of donors } \\
\text { in common }\end{array}$ & Mean NE & Mean VE \\
\hline In vivo & 145,661 & 59,153 & 1,281 & $9.31(7.29)$ & $7.75(5.98)$ \\
In vitro & 5,310 & 1,714 & & $8.07(6.08)$ & $4.13(3.51)$ \\
Heifer & 15,784 & 10,144 & 3,922 & $8.92(6.40)$ & $7.19(5.16)$ \\
Cow & 118,771 & 46,663 & & $9.44(7.42)$ & $7.80(6.09)$ \\
\hline
\end{tabular}

${ }^{1}$ Number of overlapping donors between in vivo and in vitro data sets or between heifer and cow data sets.

number of embryos produced by a donor as a heifer and as a cow, and (3) to estimate the repeatability of embryo production traits.

The data set used was an updated version to the one presented in Jaton et al. (2016). After editing, it consisted of 150,971 superovulation or OPU-IVP procedures performed on 59,586 Holstein donors between 1992 and 2016, as recorded by Holstein Canada (Brantford, ON, Canada). Two embryo production traits were available for the analyses: the total number of embryos (NE) and the number of viable embryos (VE) produced per procedure.

Information on the type of technique used for the production of embryos (in vivo or in vitro) was known for all records, as well as the service type, which indicates if the insemination was performed by either the herd owner or an AI technician when the procedure was performed in vivo. Table 1 summarizes the number of records and the number of donors considered for each type of technique. Note that only 1,281 donors had records for both types of techniques.

To determine if the donor was a heifer or a cow at embryo recovery, the data set was matched to calving records from the Canadian Dairy Network (www.cdn. ca) database. Only donors with available first calving date were kept for further analysis. Only records from 1994 and later were included in the analysis. Overall, 134,555 superovulation or OPU-IVP records from 52,885 donors were used and, from these donors, 3,922 had records both as a heifer and as a cow. A summary of the data used for the analysis of cow and heifer records is presented in Table 1.

Considering the distribution of the data, logarithmic transformation was performed to normalize the data, as explained in Jaton et al. (2016):

$$
\begin{gathered}
\mathrm{NE}_{\log }=\ln (\mathrm{NE}) \text { and } \\
\mathrm{VE}_{\log }=\ln (\mathrm{VE}+1) .
\end{gathered}
$$

The same bivariate linear model as presented in Jaton et al. (2016) was used to analyze the data using the average information-restricted maximum likelihood (AI-REML) procedure in the DMU package (Madsen and Jensen, 2008):

$$
\mathbf{y}=\mathbf{X} \boldsymbol{\beta}+\mathbf{Z}_{\mathrm{d}} \mathbf{d}+\mathbf{Z}_{\mathrm{ss}} \mathbf{s s}+\mathbf{Z}_{\mathrm{pe}} \mathbf{p e}+\mathbf{e},
$$

where $\mathbf{y}$ is a vector of logarithmic transformed observations for either $\mathrm{NE}$ or $\mathrm{VE} ; \boldsymbol{\beta}$ is a vector of systematic effects, including fixed effects of age (as linear and quadratic covariates) nested within service type, yearmonth of recovery, clinic-year of recovery, and service type; $\mathbf{d}$ is a vector of random animal additive genetic effects of the donor; ss is a vector of random animal additive genetic effects of the service sire; pe is a vector of random permanent environmental effects of the donor; $\mathbf{e}$ is a vector of random residuals; and $\mathbf{X}, \mathbf{Z}_{\mathbf{d}}, \mathbf{Z}_{\mathrm{ss}}$, and $\mathbf{Z}_{\text {pe }}$ are the corresponding incidence matrices.

Random effects were assumed to be normally distributed with means equal to zero and covariance structure equal to

$$
\operatorname{Var}\left[\begin{array}{c}
\mathbf{d} \\
\mathbf{s s} \\
\mathbf{p e} \\
\mathbf{e}
\end{array}\right]=\left[\begin{array}{cccc}
\mathbf{G}_{\mathbf{0}} \otimes \mathbf{A} & \mathbf{G}_{\mathbf{0}} \otimes \mathbf{A} & \mathbf{0} & \mathbf{0} \\
\mathbf{G}_{\mathbf{0}} \otimes \mathbf{A} & \mathbf{G}_{\mathbf{0}} \otimes \mathbf{A} & \mathbf{0} & \mathbf{0} \\
\mathbf{0} & \mathbf{0} & \mathbf{P E}_{\mathbf{0}} \otimes \mathbf{I} & \mathbf{0} \\
\mathbf{0} & \mathbf{0} & \mathbf{0} & \mathbf{R}_{\mathbf{0}} \otimes \mathbf{I}
\end{array}\right],
$$

where $\mathbf{G}_{\mathbf{0}}$ is the additive genetic (co)variance $(2 \times 2)$ matrix between traits; $\mathbf{P} \mathbf{E}_{\mathbf{0}}$ is the $(\mathrm{co})$ variance $(2 \times 2)$ matrix between traits due to permanent environmental effects; $\mathbf{R}_{\mathbf{0}}$ is the residual (co)variance $(2 \times 2)$ matrix between traits; $\mathbf{A}$ and $\mathbf{I}$ are additive relationship and identity matrices, respectively; and $\otimes$ is the Kronecker product of the respective matrices.

Heritability and repeatability (r) were calculated for the donor $(\mathrm{d})$ as

$$
\begin{gathered}
\mathrm{h}_{\mathrm{d}}^{2}=\sigma_{\mathrm{d}}^{2} /\left(\sigma_{\mathrm{d}}^{2}+2 \sigma_{\mathrm{d}, \mathrm{ss}}+\sigma_{\mathrm{ss}}^{2}+\sigma_{\mathrm{pe}}^{2}+\sigma_{\mathrm{e}}^{2}\right) \text { and } \\
\mathrm{r}_{\mathrm{d}}=\left(\sigma_{\mathrm{d}}^{2}+\sigma_{\mathrm{pe}}^{2}\right) /\left(\sigma_{\mathrm{d}}^{2}+2 \sigma_{\mathrm{d}, \mathrm{ss}}+\sigma_{\mathrm{ss}}^{2}+\sigma_{\mathrm{pe}}^{2}+\sigma_{\mathrm{e}}^{2}\right),
\end{gathered}
$$


Table 2. Genetic parameter estimates for total number of embryos (NE) and number of viable embryos (VE) for different techniques and donors (SE in parentheses; SE was not available for repeatability of the donor) ${ }^{1}$

\begin{tabular}{llccc}
\hline Item & Trait & $\mathrm{h}_{\mathrm{d}}^{2}$ & $\mathrm{~h}_{\mathrm{ss}}^{2}$ & $r_{d}$ \\
\hline In vivo & $\mathrm{NE}$ & $0.145(0.007)$ & $0.007(0.001)$ & 0.240 \\
& $\mathrm{VE}$ & $0.136(0.007)$ & $0.014(0.002)$ & 0.230 \\
In vitro & $\mathrm{NE}$ & $0.188(0.033)$ & $0.007(0.005)$ & 0.347 \\
& $\mathrm{VE}$ & $0.187(0.034)$ & $0.049(0.010)$ & 0.299 \\
Heifer & $\mathrm{NE}$ & $0.160(0.017)$ & $0.001(0.001)$ & 0.268 \\
& $\mathrm{VE}$ & $0.135(0.016)$ & $0.003(0.002)$ & 0.234 \\
Cow & $\mathrm{NE}$ & $0.158(0.008)$ & $0.009(0.001)$ & 0.251 \\
& $\mathrm{VE}$ & $0.146(0.007)$ & $0.017(0.002)$ & 0.240 \\
\hline
\end{tabular}

${ }^{1} h_{d}^{2}$ is the heritability of the donor, $h_{\mathrm{ss}}^{2}$ is the heritability of the service sire, and $r_{d}$ is the repeatability of the donor.

and for the service sire (ss) as

$$
\mathrm{h}_{\mathrm{ss}}^{2}=\sigma_{\mathrm{ss}}^{2} /\left(\sigma_{\mathrm{d}}^{2}+2 \sigma_{\mathrm{d}, \mathrm{ss}}+\sigma_{\mathrm{ss}}^{2}+\sigma_{\mathrm{pe}}^{2}+\sigma_{\mathrm{e}}^{2}\right) \text {. }
$$

Table 2 presents the genetic parameters estimated for the 2 assisted reproductive techniques for $\mathrm{NE}$ and $\mathrm{VE}$. The heritabilities estimated for the donor and service sire for the in vivo records were very similar to the ones found in our previous study (Jaton et al., 2016) and were in the same range as that reported in studies performed using an in vivo technique on Holstein donors (Asada and Terawaki, 2002; Bényei et al., 2004; König et al., 2007). On the other hand, the heritability estimates for the in vitro records were slightly higher for the donors, but similar to the estimates found in a larger study that included 18,702 OPU sessions on Holstein donors (Merton et al., 2009). Larger heritabilities were found for the service sire for VE compared with NE. Moreover, for VE, the heritability was larger for in vitro records $(0.049 \pm 0.010)$ compared with in vivo records $(0.014 \pm 0.002)$, indicating that the genetic potential of the service sire for producing embryos could influence more the number of viable embryos produced in vitro. Overall, the larger standard errors found for in vitro records are explained by the lower number of records available.

The genetic correlation between the 2 types of techniques for the donor was $0.848 \pm 0.068$ for $\mathrm{NE}$ and 0.634 \pm 0.091 for VE (Table 3), indicating a moderately high genetic correlation between the number of embryos produced in vivo and in vitro by a donor. Therefore, donors that respond well to one technique also tend to respond well to the other technique. On the service sire side, the correlation between records from the 2 techniques was not significant $(-0.266 \pm 0.299)$ for NE, but was significant $(-0.412 \pm 0.130)$ for VE (Table 3$)$. This moderate negative correlation for $\mathrm{VE}$ indicates that using the same service sire will not increase similarity between the number of viable embryos produced by the in vivo and in vitro techniques.

A Brazilian study compared the embryo production of 137 crossbred females (Bos taurus $\times$ Bos indicus) on which both in vivo and in vitro techniques were performed (Silva-Santos et al., 2014). The mean number of viable embryos of the 6 donors that produced the most embryos using an in vitro procedure was compared. The authors found no significant differences in the number of viable embryos produced by both techniques for those 6 donors, indicating that donors that responded well to IVP also responded well to superovulation. The mean number of viable embryos of the 6 donors that produced the most embryos following in vivo superovulation was also compared. A significant difference was found between the number of viable embryos produced in vivo or in vitro for the donors, indicating that donors that respond well to the in vivo technique do not necessarily respond well to the in vitro technique. The

Table 3. Genetic correlations (SE in parentheses) between techniques for total number of embryos (above the diagonal) and number of viable embryos (below the diagonal)

\begin{tabular}{|c|c|c|c|c|c|}
\hline Item & Source & \multicolumn{2}{|c|}{ In vivo } & \multicolumn{2}{|c|}{ In vitro } \\
\hline In vivo & Service sire & $-0.061(0.078)$ & & $-0.117(0.147)$ & $-0.266(0.299)$ \\
\hline \multirow[t]{2}{*}{ In vitro } & Donor & $0.634(0.091)$ & $0.151(0.139)$ & & $-0.088(0.359)$ \\
\hline & Service sire & $0.355(0.137)$ & $-0.412(0.130)$ & $0.109(0.195)$ & \\
\hline
\end{tabular}


small sample size of our study is, however, a big limitation, implying that the conclusions must be cautiously interpreted.

Genetic parameters estimated for heifer and cow donors for NE and VE are presented in Table 2. The donor heritabilities were very similar for heifers and cows, but the service sire heritability was slightly higher for cows. The heritability estimates found for the donor and service sire for the heifer and cow records are similar to the ones found in our previous study (Jaton et al., 2016) and are in the same range as reported in other studies performed on heifers (Eriksson et al., 2007), on cows (Asada and Terawaki, 2002; Bényei et al., 2004; König et al., 2007), and on heifers and cows (Merton et al., 2009). The moderate heritabilities for the donors and the near zero heritabilities for the service sire indicate that the number of embryos produced by a donor was influenced by the genetic potential of the donor, but not really by the genetic potential of the service sire. Larger standard errors were found for heritability of heifers, which can be explained by the lower number of records compared with the number of cow records.

The genetic correlations between heifer and cow records for the donor were $0.788 \pm 0.046$ and $0.723 \pm$ 0.054 for NE and VE, respectively (Table 4). These moderately high genetic correlations indicate that the number of embryos produced by donors will be similar regardless of the status (heifer or cow) of the donor at recovery and this is more so for NE. For the service sire, the genetic correlation between heifer and cow records was not significant $(0.315 \pm 0.404)$ for $\mathrm{NE}$, but was significant $(0.646 \pm 0.245)$ for VE (Table 4). Disregarding the relatively high standard error of this moderate positive correlation, it indicates that the service sire will influence in a similar manner the number of viable embryos produced by a donor either as a heifer or as a cow. Some studies have shown significant differences in embryo production traits of heifers and cows (Sartori et al., 2002; Vieira et al., 2014), but, in all cases, no donors had records as both heifers and cows.

For both NE and VE, the repeatability for the donor varied between 0.230 and 0.268 for heifer and cow records and for in vivo records. Those estimates are consistent with the ones found in our previous study (Jaton et al., 2016) and are similar to what has been previously reported in the literature (Asada and Terawaki, 2002; Bényei et al., 2004; König et al., 2007). These estimates indicate that the number of embryos produced by a donor should be slightly consistent in all superovulatory treatments performed in vivo or for a given status (heifer or cow) within an individual. For in vitro records, the repeatability estimates were slightly higher ( 0.347 for $\mathrm{NE}$ and 0.299 for $\mathrm{VE}$ ), indicating that the number of embryos produced could be more consistent within a donor if the in vitro technique is used. Overall, these repeatability estimates can be explained by the high repeatability in the number of follicles per follicular wave. A study performed on 90 beef heifers reported a very high repeatability of 0.89 for the average number of follicles per wave within an individual donor (Ireland et al., 2007). Considering that embryo production depends on the number of follicles, it makes sense that the number of embryos per procedure would also be somewhat repeatable within a donor.

In conclusion, a moderately high positive genetic correlation between the number of embryo (both NE and VE) produced in vivo and in vitro was observed, indicating that donors with high genetic potential for number of embryos produced in vivo tend also to have high potential for number of embryos produced in vitro. Similarly, the moderately high genetic correlation found between heifer and cow records indicates that a donor tend to produce a comparable number of embryos as a heifer or as a cow. Considering the estimated repeatabilities, the number of embryos should also be slightly repeatable over subsequent treatments performed on the same donor. On the other hand, the service sires seem not to play an important role on the total number of embryos produced by a donor, no matter the technique used or the status of the donor at recovery. The number of viable embryos seems to be slightly more influenced by the service sire.

The main limitations of our study were the relatively low number of donors with records for both techniques,

Table 4. Genetic correlations (SE in parentheses) between donor status for total number of embryos (above the diagonal) and number of viable embryos (below the diagonal)

\begin{tabular}{|c|c|c|c|c|c|}
\hline Item & Source & \multicolumn{2}{|c|}{ Heifer } & \multicolumn{2}{|c|}{ Cow } \\
\hline Heifer & Donor & & $0.010(0.388)$ & $0.788(0.046)$ & $-0.101(0.109)$ \\
\hline \multirow[t]{2}{*}{ Cow } & Donor & $0.723(0.054)$ & $0.005(0.243)$ & & $-0.086(0.089)$ \\
\hline & Service sire & $-0.014(0.107)$ & $0.646(0.245)$ & $0.001(0.083)$ & \\
\hline
\end{tabular}


and the low number of donors with records as both a heifer and a cow. As more data accumulates in the future, new analyses should validate the current findings.

\section{ACKNOWLEDGMENTS}

The authors are grateful to Holstein Canada (Brantford, ON, Canada) for providing the data. This study was funded by CIAQ (St-Hyacinthe, Quebec, Canada), The Semex Alliance (Guelph, Ontario, Canada), the Canadian Dairy Network (Guelph, Ontario, Canada), and the Natural Sciences and Engineering Research Council of Canada (Ottawa, Ontario, Canada).

\section{REFERENCES}

Asada, Y., and Y. Terawaki. 2002. Heritability and repeatability of superovulatory responses in Holstein population in Hokkaido, Japan. Asian-australas. J. Anim. Sci. 15:944-948.

Bényei, B., A. Gáspárdy, I. Komlósi, and A. Pécsi. 2004. Repeatability and heritability of ovulation number and embryos in damdaughters pairs in superovulated Holstein-Friesian cows. Reprod. Domest. Anim. 39:99-102.

Eriksson, S., M. Häggström, and H. Stalhammar. 2007. Genetic parameters for superovulatory response in Swedish Red Cattle and Swedish Holstein heifers. Pages 1-6 in 58th Annual Meeting of the European Association for Animal Production, Dublin, Ireland. Wageningen Academic Publishers, Wageningen, the Netherlands.

Farin, P. W., K. Moore, and M. Drost. 2007. Assisted reproductive technologies in cattle. Pages 496-508 in Large Animal Theriogenology. Saunders Elsevier, St Louis, MO

Hasler, J. F. 2014. Forty years of embryo transfer in cattle: A review focusing on the journal Theriogenology, the growth of the industry in North America, and personal reminisces. Theriogenology $81: 152-169$.
Ireland, J. J., F. Ward, F. Jimenez-Krassel, J. L. H. Ireland, G. W Smith, P. Lonergan, and A. C. O. Evans. 2007. Follicle numbers are highly repeatable within individual animals but are inversely correlated with FSH concentrations and the proportion of goodquality embryos after ovarian stimulation in cattle. Hum. Reprod. 22:1687-1695.

Jamrozik, J., J. Fatehi, G. J. Kistemaker, and L. R. Schaeffer. 2005. Estimates of genetic parameters for Canadian Holstein female reproduction traits. J. Dairy Sci. 88:2199-2208.

Jaton, C., A. Koeck, M. Sargolzaei, F. Malchiodi, C. A. Price, F. S. Schenkel, and F. Miglior. 2016. Genetic analysis of superovulatory response of Holstein cows in Canada. J. Dairy Sci. 99:3612-3623.

König, S., F. Bosselmann, U. U. von Borstel, and H. Simianer. 2007. Genetic analysis of traits affecting the success of embryo transfer in dairy cattle. J. Dairy Sci. 90:3945-3954.

Machaty, Z., J. Peippo, and A. Peter. 2012. Production and manipulation of bovine embryos: Techniques and terminology. Theriogenology 78:937-950.

Madsen, P., and J. Jensen. 2008. An User's Guide to DMU. A Package for Analyzing Multivariate Mixed Models. Version 6, release 4.7. Danish Institute of Agricultural Sciences, Tjele, Denmark.

Merton, J. S., B. Ask, D. C. Onkundi, E. Mullaart, B. Colenbrander, and M. Nielen. 2009. Genetic parameters for oocyte number and embryo production within a bovine ovum pick-up-in vitro production embryo-production program. Theriogenology 72:885-893.

Sartori, R., R. Sartor-Bergfelt, S. A. Mertens, J. N. Guenther, J. J. Parrish, and M. C. Wiltbank. 2002. Fertilization and early embryonic development in heifers and lactating cows in summer and lactating and dry cows in winter. J. Dairy Sci. 85:2803-2812.

Silva-Santos, K. C., G. Santos, C. Koetz Júnior, F. Morotti, L. Siloto, T. Marcantonio, M. Urbano, R. Oliveira, D. Lima, and M. Seneda. 2014. Antral follicle populations and embryo production - in vitro and in vivo - of Bos indicus-taurus donors from weaning to yearling ages. Reprod. Domest. Anim. 49:228-232.

Vieira, L. M., C. A. Rodrigues, M. F. Mendanha, M. F. Sá Filho, J. N. S. Sales, A. H. Souza, J. E. P. Santos, and P. S. Baruselli. 2014. Donor category and seasonal climate associated with embryo production and survival in multiple ovulation and embryo transfer programs in Holstein cattle. Theriogenology 82:204-212. 